02

\title{
Структура электронных состояний и оптические свойства соединения $\mathrm{Cr}_{80} \mathrm{Al}_{20}$
}

\author{
(С) Ю.В. Князев ${ }^{1}$, А.В. Лукоянов ${ }^{1,2}$, Ю.И. Кузьмин ${ }^{1}$, M. Vasundhara $^{3}$ \\ ${ }^{1}$ Институт фоизики металлов им. М.Н. Михеева Уральского отделения РАН, \\ 620990 Екатеринбург, Россия \\ ${ }^{2}$ Уральский федеральный университет им. Б.Н. Ельцина, \\ 620002 Екатеринбург, Россия \\ ${ }^{3}$ CSIR-National Institute for Interdisciplinary Science and Technology, Trivandrum \\ 695019 Kerala, India \\ e-mail: knyazev@imp.uran.ru
}

Поступила в редакцию 27.02.2018 г.

Выполнены эллипсометрические исследования оптических свойств интерметаллического соединения $\mathrm{Cr}_{80} \mathrm{Al}_{20}$ в спектральном интервале $0.22-15 \mu \mathrm{m}$. Проведен самосогласованный расчет электронной структуры и плотности электронных состояний данного материала. Спектр межзонного поглощения света интерпретируется на базе сравнительного анализа теоретической и экспериментальной дисперсионных зависимостей оптической проводимости. Определен ряд характеристик электронов проводимости.

DOI: $10.21883 /$ OS.2018.08.46357.58-18

\section{Введение}

Бинарные соединения хрома с алюминием $\mathrm{Cr}_{1-x} \mathrm{Al}_{x}$ вблизи стехиометрического состава $\mathrm{Cr}_{3} \mathrm{Al}$ могут упорядочиваться в различных типах кристаллических структур $\left(\mathrm{C} 11_{\mathrm{B}}, \mathrm{DO}_{3}, \mathrm{BCC}, \mathrm{X}\right.$-фаза и др.) [1], различающихся между собой целым рядом физических свойств. В частности, при переходе от одной фазы к другой могут происходить модификации в магнитном состоянии, электронной структуре, транспортных характеристиках $[1,2]$. В ряде указанных соединений, как показано в [3-5], наблюдается проявление аномальных электронных свойств, связанных с образованием энергетической щели в зонном спектре вблизи уровня Ферми. Некоторые сплавы обладают антиферромагнитным упорядочением с высокими температурами Нееля $T_{N}$ и большим магнитным моментом $\sim 1 \mu_{\mathrm{B}}$, что связано с более сильной локализацией $d$-электронов в $\mathrm{Cr}_{1-x} \mathrm{Al}_{x}$ по сравнению с чистым хромом [6] (максимальное значение $T_{N} \sim 900 \mathrm{~K}$ имеет место для соединения с $x=0.25$ [7]). Установлено, что возникновение антиферромагнитного состояния в соединениях данного типа прямым образом зависит от степени ближнего атомного порядка и структурного беспорядка [1,2]. Расчеты [3] показали, что соединения $\mathrm{Cr}_{3} \mathrm{Al}$, легированные атомами $\mathrm{Mn}$, могут проявлять свойства, характерные для сплавов Гейслера, обеспечивая высокую спиновую поляризацию электронов проводимости. В целом результаты исследований свидетельствуют, что данные материалы способны обладать ярко выраженными управляемыми свойствами, что открывает перспективу их функционального использования. В настоящей работе мы акцентируем внимание на исследовании электронной структуры соединения $\mathrm{Cr}_{80} \mathrm{Al}_{20}$. С этой целью выполнены расчеты его зонного спектра, а также проведены исследования оптических свойств в широком интервале длин волн. Результаты эксперимента обсуждаются на основе рассчитанных плотностей электронных состояний.

\section{Эксперимент и расчет}

Поликристаллические образцы бинарного $\mathrm{Cr}_{80} \mathrm{Al}_{20}$ были приготовлены из высокочистых (99.99\%) составных компонентов методом дуговой плавки в атмосфере чистого аргона. Для повышения уровня гомогенности сплава производился высокотемпературный отжиг в течение $24 \mathrm{~h}$ при $1000^{\circ} \mathrm{C}$. Данные рентгеноструктурного анализа показали, что сплав кристаллизуется в кубической структуре типа $\mathrm{DO}_{3}$, описываемой пространственной группой $\operatorname{Im} 3 m$ с параметрами решетки $a=b=c=2.9384 \AA$. Атомы хрома занимают различающиеся по симметрии позиции $(1 / 4,1 / 4,1 / 4)$ и $(0,0,0)$, атомы алюминия располагаются в кристаллографических позициях $(1 / 2,1 / 2,1 / 2)$.

Спектральные характеристики соединения определены в области длин волн $0.22 \leq \lambda \leq 15 \mu \mathrm{m} \quad(E=$ $=0.083-5.65 \mathrm{eV})$ при комнатной температуре. Эллипсометрическим методом Битти, основанным на измерении амплитуд и разностей фаз отраженных световых волн $s$ и $p$-поляризаций [8], были измерены оптические постоянные - показатели преломления $n(\lambda)$ и коэффициенты поглощения $k(\lambda)$. Измерения выполнены при одно- и двукратном отражении света от образцов в интервале углов падения $70^{\circ}-80^{\circ}$. Зеркальные поверхности 14-го класса чистоты были приготовлены механическим полированием на алмазных пастах различной зернистости.

Расчеты электронной структуры были выполнены в рамках компьютерного пакета Ouantum Expresso [9] с использованием обменно-корреляционного потенциала в 


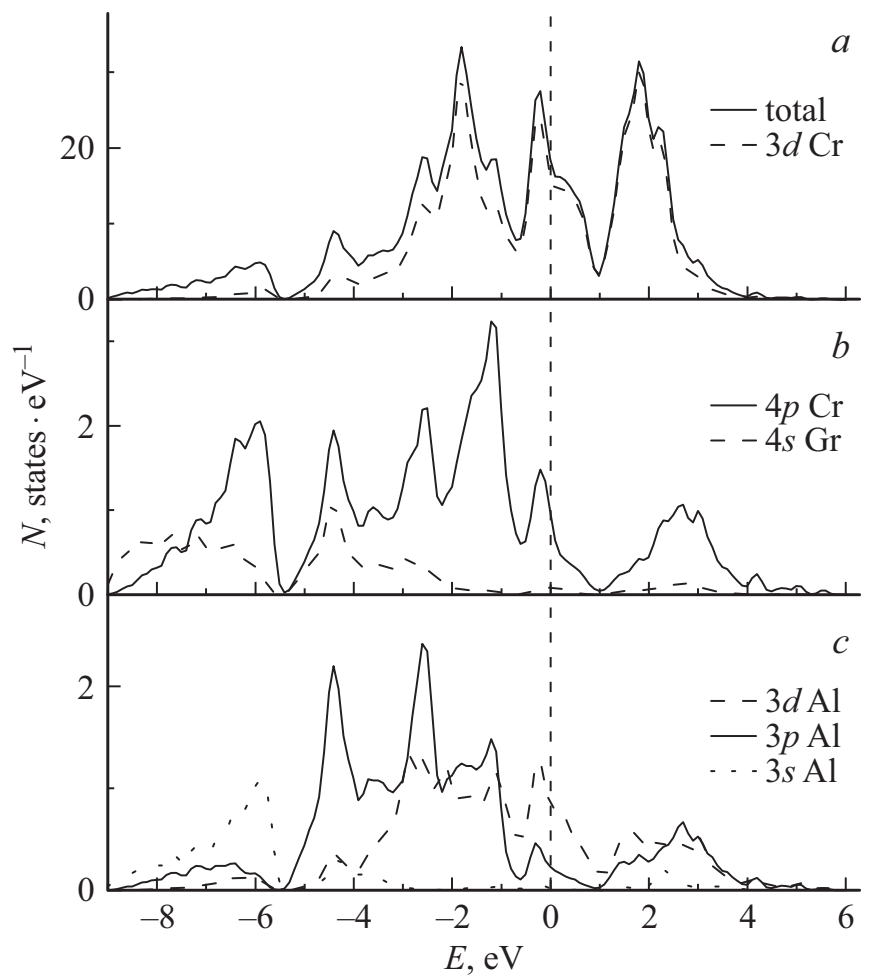

Рис. 1. Полная и парциальные (для $3 d-, 4 p$-, $4 s$-электронов $\mathrm{Cr}$ и $3 s-, 3 p-, 3 d$-электронов $\mathrm{Al}$ ) плотности состояний соединения $\mathrm{Cr}_{80} \mathrm{Al}_{20}$.

приближении обобщенной градиентной поправки GGA в версии $\mathrm{PBE}[10]$. Для проведения расчетов на базе указанной структуры была построена сверхячейка с 16 атомами. В вычислениях были использованы стандартные потенциалы. Для получения достаточной сходимости в цикле самосогласования при расчете использовался энергетический предел для плоских волн, равный $60 \mathrm{Ry}$. Интегрирование в обратном пространстве производилось по сетке из $8 \times 8 \times 8 k$-точек.

\section{Результаты и обсуждение}

На рис. 1 приведен расчет полной $N(E)$ и парциальных плотностей состояний для $3 d-, 4 p-, 4 s-$ электронов $\mathrm{Cr}$ и $3 d-, 3 p-, 3 s$-электронов Al. Спектр полной $N(E)$ состоит из трех интенсивных максимумов, основной вклад в формирование которых связан с $3 d$-электронами $\mathrm{Cr}$. Вклады в $N(E)$ от других парциальных плотностей, как показывает рис. $1, b, c$, локализованы в основном ниже энергии Ферми и имеют почти на порядок меньшую величину.

Зависимости $n(\lambda)$ и $k(\lambda)$, по значениям которых можно рассчитать ряд частотно-зависимых функций, характеризующих оптические свойства соединения $\mathrm{Cr}_{80} \mathrm{Al}_{20}$, представлены на рис. 2. Во всем спектральном интервале выполняется неравенство $k>n$, типичное для материалов с металлическим типом проводимости. При таком соотношении значений оптических постоянных действительная часть диэлектрической проницаемости $\varepsilon_{1}=n^{2}-k^{2}$ является отрицательной величиной, что также характерно для проводящих сред. Немонотонное поведение этих параметров в коротковолновом диапазоне при $\lambda<\sim 1.5 \mu \mathrm{m}$ связано с межзонным поглощением света. В свою очередь постепенный рост $n$ и $k$ при более высоких значениях $\lambda$ указывает на внутризонный механизм взаимодействия электромагнитных волн с электронами проводимости. На вставке рис. 2 приводится дисперсия отражательной способности $R=\left[(n-1)^{2}+k^{2}\right] /\left[(n+1)^{2}+k^{2}\right]$ соединения. Поведение энергетической зависимости этого параметра также характеризуется немонотонной структурой, а при уменьшении частоты света значение $R$ растет, приближаясь к единице.

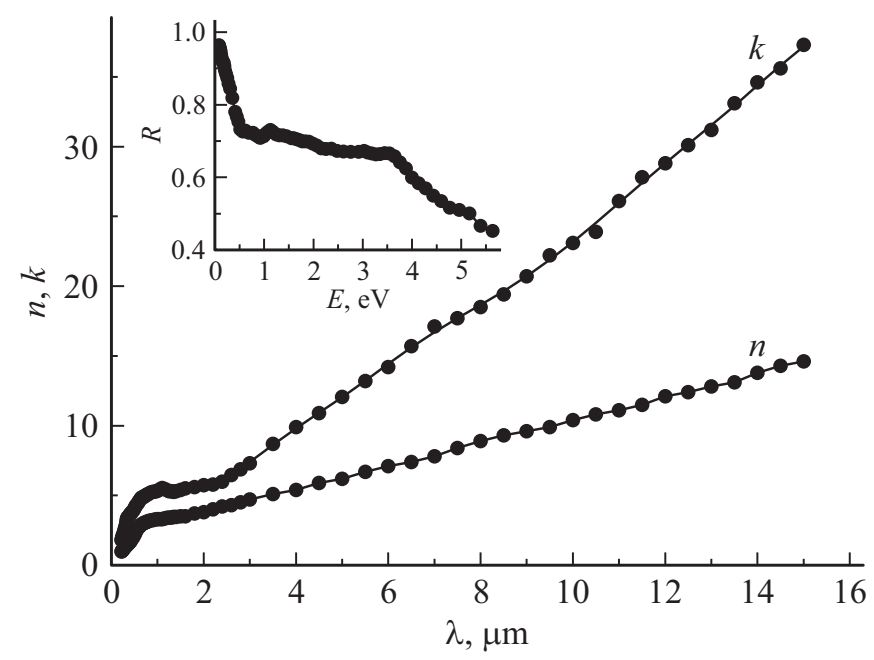

Рис. 2. Дисперсия оптических постоянных $n, k$ и отражательной способности $R$ (вставка) соединения $\mathrm{Cr}_{80} \mathrm{Al}_{20}$.

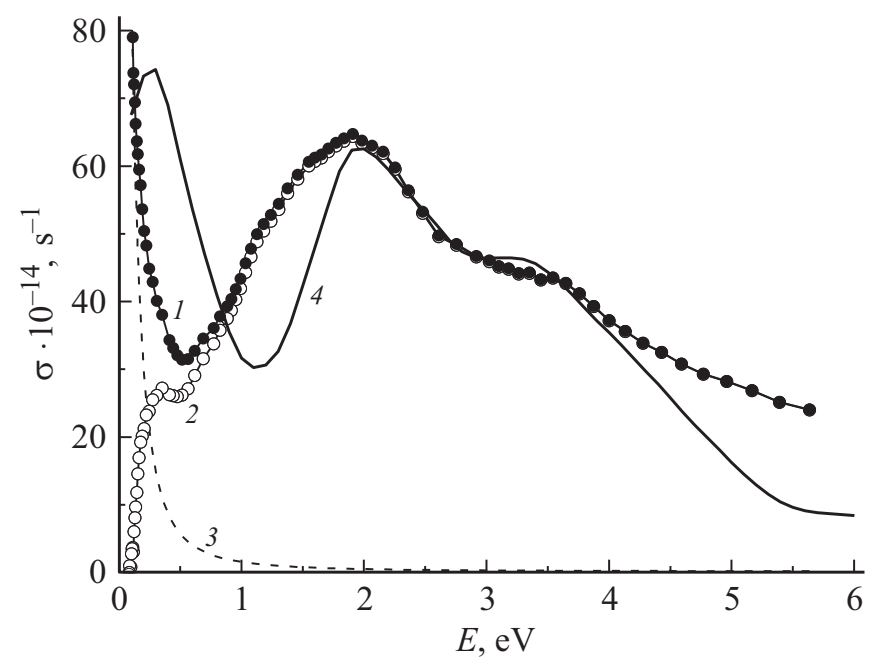

Рис. 3. Оптическая проводимость соединения $\mathrm{Cr}_{80} \mathrm{Al}_{20}$. 1 - эксперимент, 2 - вклад межзонного поглощения, 3 друдевский вклад, $4-$ расчет из полной плотности состояний. 
На рис. 3 темными точками представлена экспериментальная частотная зависимость оптической проводимости исследуемого соединения $\sigma(\omega)=n k \omega / 2 \pi(\omega-$ частота света). Данный параметр характеризует частотную зависимость и интенсивность оптического отклика отражающей среды и в отличие от статической проводимости определяется не плотностью состояний на уровне Ферми, а особенностями поведения $N(E)$ во всем исследуемом интервале энергий световых квантов. В структуре дисперсионной зависимости $\sigma(\omega)$ отчетливо проявляются два интервала, соответствующие различным типам взаимодействия электронов с электромагнитной волной. В низкочастотной (инфракрасной) области поведение $\sigma(\omega)$ соответствует внутризонному (друдевскому) механизму возбуждения свободных электронов квантами света малых энергий $\left(\sigma \sim 1 / \omega^{2}\right)$ и характеризуется параметрами носителей тока - плазменной $\omega_{\mathrm{P}}$ и релаксационной $\gamma$ частотами электронов проводимости. Эти параметры могут быть определены из классических соотношений Друде с помощью оптических постоянных, измеренных в инфракрасном диапазоне. Частота релаксации является аддитивной величиной, учитывающей все типы рассеяния электронов в процессе их возбуждения электромагнитной волной, и в пределе $\omega \rightarrow 0$ пропорциональна статической проводимости. Плазменная частота, характеризующая коллективные осцилляции валентных электронов и зависящая от эффектов межэлектронной корреляции [11], определяется соотношением $\omega_{\mathrm{P}}^{2}=4 \pi N e^{2} / m$, где $N$, e и $m-$ концентрация, заряд и масса электронов. В длинноволновой области спектра эти характеристики стабилизируются при значениях $\gamma=1.2 \cdot 10^{14} \mathrm{~s}^{-1}$ и $\omega_{\mathrm{P}}=5.9 \cdot 10^{15} \mathrm{~s}^{-1}$. Концентрация электронов проводимости при этом равна $N=1.1 \cdot 10^{22} \mathrm{~cm}^{-1}$.

При $E>\sim 0.5 \mathrm{eV}$ форма зависимости $\sigma(\omega)$ указывает на доминирующую роль межзонного поглощения. Дисперсия оптической проводимости в этом диапазоне характеризуется широкой асимметричной абсорбционной полосой с максимумом вблизи $2 \mathrm{eV}$ и высокоэнергетическим „плечом“ при $2.5-3.5 \mathrm{eV}$. Данная структура формируется электронными переходами между энергетическими состояниями, разделенными уровнем Ферми, a ее форма определяется особенностями зонной структуры соединения $\mathrm{Cr}_{80} \mathrm{Al}_{20}$. Природа образования указанной полосы межзонного поглощения может быть объяснена на основе рассчитанной плотности электронных состояний. С этой целью мы проводим сравнение межзонной оптической проводимости $\sigma_{\mathrm{м} 3}(\omega)=\sigma(\omega)-\sigma_{\mathrm{D}}\left(\sigma_{\mathrm{D}}-\right.$ друдевский вклад), полученной из эксперимента, с соответствующей теоретической зависимостью, вычисленной на основе полной $N(E)$ (рис. 1$)$. Расчет $\sigma_{\mathrm{M}}(\omega)$, реализованный по методу [12] на основе свертки плотностей состояний ниже и выше $E_{\mathrm{F}}$, позволяет качественно оценить суммарный вклад всех типов межзонных переходов в оптическую проводимость при условии их равной вероятности. Результат такого расчета показан на рис. 3 совместно с экспериментальным спектром $\sigma_{\text {мз }}(\omega)$.
При сравнении экспериментальной и теоретической зависимостей можно отметить как определенное сходство (совпадение энергий локализации максимумов и „плечей“6), так и различия, проявляющиеся в основном в соотношении интенсивностей максимумов и различии положения низкоэнергетического склона полосы. В целом зонные расчеты, проведенные для данного соединения, адекватно отражают наблюдаемую картину межзонного оптического поглощения, предсказывая максимумы при $0.4,2 \mathrm{eV}$, а также „плечо“ на высокоэнергетическом склоне. При этом положение и природа главных пиков в плотности электронных состояний позволяет объяснить возникновение максимумов в экспериментальной кривой $\sigma_{\text {мз }}(\omega)$. Их формирование, как следует из структуры $N(E)$, связано с межзонными переходами между состояниями, связанными с $3 d$-электронами $\mathrm{Cr}$. Так, образование низкоэнергетического максимума при $0.4 \mathrm{eV}$ обусловлено переходами между состояниями указанного типа, образующими широкий пик и разделенными уровнем Ферми. Протяженная структура в $\sigma_{\text {мз }}(\omega)$ с максимумом при $\sim 2 \mathrm{eV}$ формируется за счет электронных переходов в $3 d$-зонах хрома, локализованных соответственно при $1-3 \mathrm{eV}$ ниже $E_{\mathrm{F}}$ и непосредственно выше $E_{\mathrm{F}}$. При этом образование „Плеча“ ассоциируется с возбуждением электронов $\mathrm{Cr} 3 d$-типа с состояний, расположенных в интервале $0-0.5 \mathrm{eV}$ ниже $E_{\mathrm{F}}$, в состояния, находящиеся в пределах $1-3 \mathrm{eV}$ выше $E_{\mathrm{F}}$. Другие типы переходов дают существенно меньшие вклады, что обусловлено малостью значений их парциальных плотностей состояний. Следует отметить, что ввиду качественного характера вычислений $\sigma_{\text {мз }}(\omega)$, проведенного без учета вероятностей квантовых переходов, в деталях тонкой структуры теоретической и экспериментальной зависимостей нет полного совпадения. Например, величина расчетной $\sigma_{\text {мз }}(\omega)$ в низкоэнергетическом интервале, где предсказывается интенсивный максимум при $0.4 \mathrm{eV}$, оказывается существенно завышенной.

\section{Заключение}

В работе впервые были исследованы оптические свойства и электронная структура соединения $\mathrm{Cr}_{80} \mathrm{Al}_{20}$. B самосогласованном расчете, проведенном методом LDA, рассчитаны полные и парциальные плотности электронных состояний в интервале нескольких электронвольт от уровня Ферми. Эллипсометрическим методом в широком интервале спектра измерены оптические характеристики данного материала. На основе результатов расчета проведена интерпретация экспериментальной зависимости оптической проводимости в области квантового поглощения света. Показано, что поведение частотной зависимости этого параметра качественно воспроизводится в рамках представленного расчета. Локализация и энергетическая протяженность основных структурных особенностей на опытной кривой оптической проводимости согласуется с соответствующей 
теоретической зависимостью. Из спектральных данных определены характеристики электронов проводимости: концентрация, плазменная и релаксационная частоты.

Работа выполнена в рамках государственного задания ФАНО России (тема „Электрон“, № ААААА18-118020190098-5) при частичной поддержке РФФИ (проект № 17-52-45056). А.В. Лукоянов благодарит за поддержку Правительство Российской Федерации (постановление № 211 контракт № 02.А03.21.0006).

\section{Список литературы}

[1] Boekelheide Z., Saerbeck T., Stampfl A.P.J., Robinson R.A., Stewart D.A., Hellman F. // Phys. Rev. B. 2012. V. 85. P. 094413. doi 10.1103/PhysRevB.85.094413

[2] Boekelheide Z., Stewart D.A., Hellman F. // Phys. Rev. B. 2012. V. 86. P. 085120. doi 10.1103/PhysRevB.86.085120

[3] Li J., Chen H., Li Y., Xiao Y., Li Z. // J. Appl. Phys. 2009. V. 105. P. 083717. doi $10.1063 / 1.3116533$

[4] Gao G.Y., Yao K.L. // Appl. Phys. Lett. 2013. V. 103. P. 232409. doi $10.1063 / 1.4840318$

[5] Boekelheide Z., Gray A.X., Papp C., Balke B., Stewart D.A., Ueda S., Kobayashi K., Hellman F., Fadley C.S. // Phys. Rev. Lett. 2010. V. 105. P. 236404. doi 10.1103/PhysRevLett.105.236404

[6] Costa M.M.R., Brown P.J. // J. Phys. F: Met. Phys. 1977. V. 7. N 3. P. 493. doi 10.1088/0305-4608/7/3/019

[7] Fawcett E. // Rev. Mod. Rhys. 1988. V. 60. N 1. P. 209. doi 10.1103/RevModPhys.60.209

[8] Beattie J.R., Conn G.K.T. // Phil. Mag. 1955. V. 46. P. 222. doi 10.1080/14786440208520565

[9] Giannozzi P., Baroni S., Bonini N., Calandra M., Car R., Cavazzoni C., Ceresoli D., Chiarotti G.L., Cococcioni M., Dabo I., Dal Corso A., de Gironcoli S., Fabris S., Fratesi G., Gebauer R., Gerstmann U., Gougoussis C., Kokalj A., Lazzeri M., Martin-Samos L., Marzari N., Mauri F., Mazzarello R., Paolini S., Pasquarello A., Paulatto L., Sbraccia C., Scandolo S., Sclauzero G., Seitsonen A.P., Smogunov A., Umari P., Wentzcovitch R.M. // J. Phys.: Condens. Matter. 2009. V. 21. P. 395502. doi 10.1088/09538984/21/39/395502

[10] Perdew J.P., Burke K., Ernzerhof M. // Phys. Rev. Lett. 1996. V. 77. N 18. P. 3865. doi 10.1103/PhysRevLett.77.3865

[11] Каганов М.И., Слезов В.В. // ЖЭТФ. 1957. Т. 32. № 6. C. 1496; Kaganov M.I., Slezov V.V. // JETP. 1957. V. 5. N 6. P. 1216.

[12] Knyazev Yu.V., Lukoyanov A.V., Kuz'min Yu.I., Kuchin A.G., Nekrasov I.A. // Phys. Rev. B. 2006. V. 73. P. 094410. doi 10.1103/PhysRevB.73.094410 